\title{
PERFIL SOCIODEMOGRÁFICO E CLÍNICO DE PACIENTES COM INCONTINÊNCIA URINÁRIA - UM ESTUDO DE CASO EM MARINGA- PR
}

Kátia Cristina de Souza ${ }^{1}$; Ayanne Rodrigues Cambiriba ${ }^{2}$; Mateus Dias Antunes ${ }^{3}$; Sonia Maria Marques Gomes Bertolini ${ }^{4}$.

${ }^{1}$ Fisioterapeuta pelo Centro Universitário de Maringá - UNICESUMAR - Maringá, Paraná, Brasil.

${ }^{2}$ Acadêmica do Curso de Fisioterapia do Centro Universitário de Maringá, UNICESUMAR - Maringá, Paraná, Brasil.

${ }^{3}$ Mestrando em Promoção da Saúde do Centro Universitário Cesumar UNICESUMAR - Maringá, Paraná, Brasil. Bolsista CAPES.

mateus antunes03@hotmail.com

${ }^{4}$ Professora, doutora do Programa de Mestrado em Promoção da Saúde do Centro Universitário de Maringá - UNICESUMAR - Maringá, Paraná, Brasil.

Recebido em: 03/10/2016 - Aprovado em: 21/11/2016 - Publicado em: 05/12/2016 DOI: 10.18677/EnciBio 2016B 137

\begin{abstract}
RESUMO
A falta de continência urinária, caracterizada pela perda involuntária de urina, acomete a qualidade de vida dos portadores, afetando o contato social, a higiene e a vida sexual. Assim, fazem se necessários estudos sobre o perfil sociodemográfico e clínico de pacientes acometidos por essa disfunção. O objetivo deste estudo foi analisar o perfil sociodemográfico e clínico de pacientes com incontinência urinária atendidos no setor de Fisioterapia Uroginecológica de uma clínica escola do noroeste do Paraná. Trata-se de um estudo descritivo, com dados secundários coletados de prontuários de 2013 a 2015. A maior ocorrência de incontinência urinária foi encontrada na faixa etária de 66 a 75 anos. Verificou-se que $66,67 \%$ dos participantes apresentaram Incontinência Urinária Mista, 21,21\% Incontinência Urinária de Esforço e 12,12\% Incontinência Urinária de Urgência. A maior prevalência de incontinência urinária nos pacientes da Clínica Escola foi encontrada no sexo feminino, em idosos, sendo que o tipo misto foi o de maior ocorrência, embora com relatos de perda com intensidade leve.
\end{abstract}

PALAVRAS-CHAVE: Assoalho pélvico, Incontinência Urinária, Perfil de Saúde.

\section{DEMOGRAPHIC AND CLINICAL PROFILE OF PATIENTS WITH URINARY INCONTINENCE}

\begin{abstract}
The lack of urinary continence, characterized by involuntary loss of urine, affects the quality of life of disabled people, affecting the social contact, hygiene and sex life. So does if necessary studies on the socio-demographic and clinical profile of patients affected by this dysfunction. The objective of the present study was to analyze the demographic and clinical profile of patients with urinary incontinence met in the sector of Physiotherapy Urogynecological a clinical School of the Northwest of
\end{abstract}


Paraná. This is a descriptive study, with secondary data collected from medical records, in the period from 2013 to 2015. The higher incidence of urinary incontinence was found at the age of 66 to 75 years. It turned out that $66.67 \%$ of the participants showed mixed Urinary Incontinence $21.21 \%$ stress urinary incontinence and urinary Urgency Incontinence $12.12 \%$. The higher prevalence of urinary incontinence in patients of the clinic school was found in the female, in the elderly, and the mixed type was the higher incidence, although with reports of loss with light intensity.

KEYWORDS: Pelvic Floor, Urinary Incontinence, Health Profile.

\section{INTRODUÇÃO}

Qualquer queixa de perda involuntária de urina é considerada Incontinência Urinária (SAMUELSSON et al., 2015). Entretanto, essa definição não leva em consideração a grande variação desse sintoma e a interferência causada na vida dos indivíduos incontinentes (ARAÚJO et al., 2015). A incontinência urinária (IU) pode ser classificada em quatro grupos: Incontinência Urinária de Esforço (IUE) definida como perda involuntária de urina ao esforço ou exercício, ou ao espirrar ou tossir; Incontinência Urinária de Urgência (IUU) sendo entendida como a perda involuntária de urina acompanhada, ou imediatamente precedida, por urgência de urinar; Incontinência Urinária Mista (IUM), disfunção que apresenta associação dos sintomas da IUE e IUU; e Incontinência Urinária Inconsciente (IUI) que é a perda de urina sem urgência ou reconhecimento consciente do extravasamento (CAVALCANTE et al., 2014; SUMARDI et al., 2016; PARK \& HONG, 2016).

A IU é frequentemente de origem multifatorial (DEDICAÇÃO et al., 2009), tais como idade avançada, paridade, parto vaginal, queda dos níveis de estrogênio na menopausa, ser do sexo feminino, tratamento do câncer de próstata e incapacidades físicas e mentais (FIGUEIREDO et al., 2008). A prevalência de IU varia conforme os estudos publicados, fato este pode ser decorrente das diferenças metodológicas utilizadas e tipos de populações (SILAY et al., 2016).

No Brasil são poucos os estudos atuais sobre a prevalência de IU. A estimativa varia entre $20 \%$ e $35 \%$ para mulheres entre 50 e 75 anos. No entanto, somente $25 \%$ a $50 \%$ procuram atendimento médico (JEREZ-ROING et al., 2016; SMITH, 2016). Contudo, a porcentagem de mulheres que procuram assistência médica com queixa de perda de urina e a porcentagem das que têm perda de urina regular ou esporadicamente têm se mostrado divergentes (EL-AZAB \& SHAABAN, 2010).

Embora com alta prevalência, pouco mais da metade dos pacientes consultam seus médicos a respeito do problema por considerá-lo decorrente da vida e sintoma natural. A IU só ganha importância quando influencia na qualidade de vida, devido aos problemas progressivos de ordem higiênica, social e psicológica (SAMUELSSON et al., 2015). É mais comum em mulheres por apresentarem menor comprimento uretral e maior chance de injúrias deste compartimento durante a gestação e o parto. A IU e sua severidade aumentam com a idade (MARQUES et al., 2015).

Atualmente, há várias opções terapêuticas para incontinência, incluindo intervenções comportamentais, técnicas de reabilitação da musculatura do assoalho pélvico, tratamento fisioterapêutico, procedimentos cirúrgicos e medicamentosos 
(VITALE et al., 2016). No entanto, a melhor forma de intervir é identificando as condições de ocorrência da IU, sendo assim, o objetivo deste estudo foi caracterizar o perfil sociodemográfico e clínico de pacientes com incontinência urinária atendidos em uma clínica escola, bem como verificar os possíveis fatores associados.

\section{MATERIAL E MÉTODOS}

O presente estudo foi realizado na Clínica de Fisioterapia do Centro Universitário de Maringá (UNICESUMAR), na cidade de Maringá, região Noroeste do Paraná. Considerando os aspectos éticos da pesquisa, o projeto foi aprovado pelo Comitê de Ética e Pesquisa Envolvendo Seres Humanos do Centro Universitário Cesumar - UNICESUMAR conforme parecer n. 850.002/2014.

O estudo tem por característica ser do tipo descritivo, com abordagem quantitativa, com pesquisa de dados secundários, realizada por meio de análise documental. Os dados foram coletados de 33 prontuários, dentre estes, 26 do sexo feminino e 7 masculino e pacientes com diagnóstico de IU, de 2013 a 2015, atendidos no setor de Fisioterapia Uroginecológica da Clínica Escola de Fisioterapia do UNICESUMAR, os quais atendiam os critérios de inclusão: prontuários de pacientes com diagnóstico de IU independente do tipo, do período de 2013 a 2015; ambos os sexos; pacientes de todas as idades.

O instrumento de coleta dos dados foi à própria ficha de avaliação utilizada no setor, não sendo observada qualquer alteração no modelo da ficha durante os anos compreendidos pela pesquisa. A ficha de avaliação apresentava diversas informações, tais como: dados pessoais, anamnese, queixa principal, quando surgiu o problema, circunstância em que ocorre a perda, frequência da perda, frequência da micção, quantidade da perda, estilo de vida, convívio social, uso de proteção, antecedentes ginecológicos, antecedentes obstétricos, antecedentes ano-retais, atividade sexual, tratamentos anteriores, diagnóstico fisioterapêutico.

Para caracterizar o tipo de IU apresentada pelos pacientes, utilizaram-se como parâmetro as ocasiões em que ocorre a perda, segundo a classificação ICIQSF (International Consultation on Incontinence Questionnaire - Short Form), validada e traduzida para o Brasil por TAMANINI et al., (2004). Foi utilizada essa metodologia, pois muitas fichas de avaliação utilizadas para este estudo eram conflitantes nessa questão. Deve-se salientar ainda, que as ocasiões de ocorrência da perda não foram avaliadas por exames urodinâmicos.

As informações contidas na ficha de avaliação viabilizaram a obtenção dos dados pertinentes ao estudo. Os dados obtidos foram tabulados no Microsoft Excel e analisados utilizando estatística descritiva (frequência relativa e absoluta para as variáveis categóricas e médias e desvio padrão para as variáveis numéricas).

\section{RESULTADOS E DISCUSSÃO}

A maioria dos pacientes do setor uroginecológico de fisioterapia era do sexo feminino (78,79\%), encontrava-se na faixa etária entre 66 e 75 anos $(61,70 \pm 13,59)$ e $69,70 \%$ eram casados (Tabela 1 ). 
TABELA 1. Características sociodemográficas dos pacientes com incontinência urinária atendidos no setor de fisioterapia uroginecológica da Clínica de Fisioterapia do Centro Universitário de Maringá, no período de 2013 a 2015.

\begin{tabular}{lcc}
\hline VARIAVËIS & N & $\%$ \\
\hline Sexo & 26 & 78,79 \\
Feminino & 07 & 21,21 \\
Masculino & & \\
Idade & 01 & 3,03 \\
$15-25$ anos & - & - \\
$26-35$ anos & 02 & 6,06 \\
$36-45$ anos & 06 & 18,18 \\
$46-55$ anos & 09 & 27,27 \\
$56-65$ anos & 13 & 39,40 \\
$66-75$ anos & 02 & 6,06 \\
+76 anos & & \\
Estado Civil & 23 & 69,70 \\
Casado & 03 & 9,09 \\
Divorciado & 03 & 9,09 \\
Solteiro & 04 & 12,12 \\
Viúvo & & \\
Profissão & 11 & 33,33 \\
Aposentado & 11 & 33,33 \\
Do lar & 02 & 6,06 \\
Doméstico & 27,27 \\
Outras profissões & 09 & \\
& & \\
\hline
\end{tabular}

No presente estudo, de acordo com o sexo, a prevalência de IU foi maior no sexo feminino. Baseado nos estudos científicos desenvolvidos na maioria dos países, a IU tem uma elevada prevalência (SMITH, 2016), principalmente entre mulheres (SANTOS \& SANTOS, 2010).

A maioria de IU nas mulheres apontada na literatura ocorre devido à diferença do tamanho da uretra entre os sexos masculino e feminino, a anatomia do assoalho pélvico, as decorrências da gestação e do parto nos mecanismos de continência e as alterações hormonais, caracterizadas pelo esgotamento dos folículos ovarianos e hipoestrogenismo progressivo (CARRARA et al., 2012). Esse fenômeno pode ser explicado pela ocorrência das alterações orgânicas ocorridas na senescência que podem ser potencializadas por características típicas ao gênero feminino, como a história reprodutiva que expõe o sistema geniturinário a maiores traumas e pelas alterações hormonais decorrentes do climatério (WAETJEN et al., 2008).

A prevalência de IU masculina varia de 3 a $39 \%$, conforme a faixa etária. Entre os principais fatores de risco para o desenvolvimento de IU no homem encontram-se: a idade, os sintomas do sistema urinário, as infecções urinárias, as doenças neurológicas, os déficits cognitivos, funcionais e de mobilidade e as cirurgias do sistema urinário, sobretudo as prostatectomias (ARAÚJO et al., 2015). No presente estudo, a prevalência de IU nos homens encontra-se dentro da faixa etária verificada em estudos.

A respeito do tipo de IU, 66,67\% dos participantes apresentaram IUM, 21,21\% IUE e 12,12\% IUU. Em relação à frequência da perda de urina (Tabela 2), a maioria 
$(42,42 \%)$ relatou que a perda ocorre "quase sempre", sendo uma perda de intensidade leve $(39,39 \%)$.

Em relação à idade, a média encontrada foi de $61,70 \pm 13,59$ anos, corroborando com o estudo de ARAÚJO et al., (2010) que a faixa etária mais atingida dentre a população idosa foi de 60 a 69 anos. No presente estudo notou-se o aumento da prevalência de IU conforme o aumento da idade. A IU e sua severidade aumentam com a idade (MARQUES et al., 2015), considerado também esse fato em outro estudo de SOUZA et al., (2015). Os resultados deste estudo, com relação ao estado civil, revelaram que a maioria $(70 \%)$ dos pacientes atendidos no setor uroginecológico da clinica de fisioterapia eram casados, o que corrobora com os achados do estudo de KNORST et al. (2011).

Quanto à profissão dos participantes, estes ou estavam aposentados (33,33\%) ou eram do lar $(33,33 \%)$. Entretanto, não foram encontrados na literatura dados semelhantes. Por outro lado, CARRARA et al., (2012) realizaram um estudo que encontrou a renda familiar mensal de mulheres incontinentes entre um e dois salários mínimos, o que condiz com as profissões encontradas neste estudo. Os fatores econômicos afetam a qualidade de vida das mulheres com IU, dificultando os cuidados com a saúde e a adesão ao tratamento adequado. No entanto, é importante saber quais os tipos de profissões esses pacientes já exerceram no decorrer da vida, já que algumas profissões podem predispor o indivíduo a desenvolver IU.

Como ilustrado na figura 1, no que se refere ao do tipo de IU a maior prevalência encontrada foi de IUM (67\%). Quanto à estratificação do tipo de IU, outros estudos encontraram dados semelhantes, sendo que a maior prevalência foi de IUM (SANTOS \& SANTOS, 2010; KNORST et al., 2011) seguida de IUE e de IUU (LEROY et al., 2012).

\section{TIPO DE INCONTINÊNCIA}

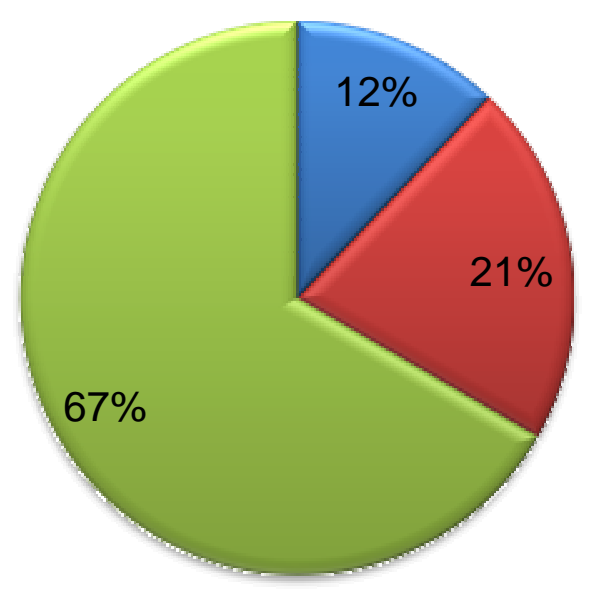

- Incontinência Urinária de Urgência

- Incontinência Urinária de Esforço

$\checkmark$ Incontinência Urinária Mista

FIGURA 1. Distribuição dos casos de incontinência urinária em pacientes atendidos no setor de fisioterapia uroginecológica da Clínica de Fisioterapia do Centro Universitário de Maringá, no período de 2013 a 2015.

O diagnóstico da IU não é tão complexo. A presença é confirmada quando há qualquer queixa de perda involuntária de urina (LOPES et al., 2009). No entanto, o 
diagnóstico que determina o tipo de IU, que o individuo apresenta, pode ser difícil de ser estabelecido em alguns casos. Portanto para a investigação dessa condição, utilizam-se os seguintes recursos: anamnese, questionários de qualidade de vida, diário urinário, pad test, exame físico, estudo urodinâmico, sendo que este último pode permitir diagnóstico preciso (PARK \& HONG, 2016). No presente estudo, apenas foi realizada a anamnese, pois é de fácil aplicabilidade. Uma anamnese bem realizada associada a um exame físico minucioso muitas vezes é capaz de proporcionar o diagnóstico preciso e orientar o tratamento correto (BORGES et al., 2010).

Em relação à frequência da perda, $43 \%$ dos pacientes relataram perder urina quase sempre, seguida de $30 \%$ que perdem esporadicamente e $27 \%$ que disseram perder sempre (Tabela 2). Ao analisar o uso de protetores relacionados à frequência da perda foi verificado que a frequência da perda não apresenta relação com o uso ou não de protetor, já que mesmo entre as pessoas que perdem urina esporadicamente a utilização de protetores é grande. Verificou-se que entre os que perdem urina sempre $67 \%$ deles usam protetores. Nos que perdem quase sempre $71 \%$ utilizam protetores. Já, entre os que perdem esporadicamente $70 \%$ usam protetores.

Algumas mulheres que perdem urina involuntariamente optam por usar um produto absorvente para a incontinência quando estão em situações de maior risco, como sair de casa, fazer compras, atividade física, viagens entre outras que podem causar essa situação. A perda da qualidade de vida está relacionada à gravidade da IU, e que essa, por sua vez, está associada com a idade do paciente. A gravidade do problema pode ser medida através da frequência dos episódios da perda urinária, ou ainda através de um índice de gravidade no qual são realizadas algumas perguntas ao paciente (SILAY et al., 2016).

TABELA 2. Distribuição da frequência da perda de urina em pacientes atendidos no setor de fisioterapia uroginecológica da Clínica de Fisioterapia do Centro Universitário de Maringá, no período de 2013 a 2015.

\begin{tabular}{lcc}
\hline FREQUÊNCIA DA PERDA & N & $\%$ \\
\hline Sempre & 09 & 27,27 \\
Quase sempre & 14 & 42,42 \\
Esporadicamente & 10 & 30,30 \\
\hline
\end{tabular}

A respeito da intensidade da perda de urina $40 \%$ dos pacientes (TABELA 3) relataram que têm perda leve, $30 \%$ contaram ter uma perda moderada, na mesma proporção dos que têm uma perda intensa (30\%). Pode se dizer, também, que a intensidade da perda está diretamente relacionada ao uso de protetores (absorventes, fraldas e outros), sendo que o uso deste recurso aumentou conforme o grau de severidade relacionado com a perda. Constatou-se que entre os que têm perda leve, $54 \%$ utilizam proteção; nos com perda moderada $70 \%$ utilizam esse recurso e os que têm perda intensa $90 \%$ lançam mão do uso de protetores.

TABELA 3. Distribuição da intensidade de perda de urina em pacientes atendidos no setor de fisioterapia uroginecológica da Clínica de Fisioterapia do Centro Universitário de Maringá, no período de 2013 a 2015.

\begin{tabular}{lcc}
\hline INTENSIDADE DA PERDA & $\mathbf{N}$ & $\%$ \\
\hline & & \\
Leve & 13 & 39,39 \\
Moderada & 10 & 30,30 \\
Intensa & 10 & 30,30 \\
\hline
\end{tabular}


No estudo de SILVA \& D’ELBOUX (2012) com relação às características da IU, houve predomínio $(61,5 \%)$ do relato de perda urinária diversas vezes ao dia e em pequena quantidade. Estudos mostram pequena relação entre o volume de urina perdido e o sofrimento do paciente. O grau de perturbação de cada pessoa pela IU é influenciado por diversos fatores, que incluem valores culturais e expectativas acerca de continência e incontinência. Com frequência há discordância entre a intensidade objetiva dos sintomas e o incômodo subjetivo (MORRISROE et al., 2014).

Foi verificado que a intensidade da perda pode interferir no estilo de vida do paciente. Quanto mais intensa a perda, mais impacto no estilo de vida haverá. Segundo os dados analisados, verificou-se que dos que apresentam uma perda leve $62 \%$ são sedentários e nos que tem uma perda moderada, $60 \%$ são sedentários. Acrescenta-se que entre os que relataram ter uma perda intensa, $80 \%$ são sedentários. Ao analisar a relação entre o estilo de vida e a frequência da perda pode se dizer que não há relação entre essas variáveis, já que a porcentagem de sedentários é praticamente a mesma em todas as categorias (sempre, quase sempre e esporadicamente).

A IU pode impactar negativamente na qualidade de vida dos indivíduos com essa queixa, interferindo diretamente nas atividades diárias dos idosos, de tal modo com que haja um ciclo vicioso de sofrimento e ansiedade, além de um sentimento de vergonha, os levam a um importante incômodo psicológico e variado grau de isolamento social (PEDRO et al., 2011).

Conforme tabela 4 , no que se refere ao estilo de vida, grande parte dos pacientes $(66,67 \%)$ eram sedentários, sendo que $63,64 \%$ com convívio social normal, e $69,70 \%$ dos participantes utilizavam protetores. No que diz respeito aos antecedentes obstétricos, das 26 mulheres participantes do estudo a média de número de partos foi de $2,77( \pm 1,92)$, sendo que $68,18 \%$ delas tiveram todos os partos normais. Menos da metade $(42,31 \%)$ das mulheres apresentaram prolapsos e destas, $81,82 \%$ realizaram cirurgia para reconstrução do períneo. Entre os homens, 6 (85,71\%) tiveram neoplasias de próstata, sendo que $100 \%$ destes passaram por prostatectomia e desenvolveram IU após esse procedimento. No que diz respeito à vida sexual, $66,67 \%$ dos participantes estão inativos.

TABELA 4 - Perfil clínico dos pacientes atendidos no setor de fisioterapia uroginecológica da Clínica de Fisioterapia do Centro Universitário de Maringá, no período de 2013 a 2015.

\begin{tabular}{lcc}
\hline VARIAVÉIS & N & $\%$ \\
\hline Estilo de vida & & \\
Sedentário & 22 & 66,67 \\
Ativo & 11 & 33,33 \\
Convívio Social & 21 & 63,64 \\
Normal & 10 & 30,30 \\
Diminuído & 02 & 6,06 \\
Ausente & & \\
Uso de proteção & 23 & 69,70 \\
Sim & 10 & 30,30 \\
Não & & \\
Antecedentes Obstétricos & & \\
Número de partos & 04 & 15,38 \\
Nenhum parto & 01 & 3,85 \\
1 parto & 08 & 30,77 \\
2 partos & &
\end{tabular}

ENCICLOPÉDIA BIOSFERA, Centro Científico Conhecer - Goiânia, v.13 n.24; p. 14892016 
5 partos

+ 5 partos

Tipo de parto

Normal

68,18

Cesárea

Normal/Cesárea

Alterações Uroginecológicas

Prolapsos

42,31

Neoplasias prostáticas

85,71

Cirurgia

Reconstrução de períneo

Prostatectomia

100,00

Atividade Sexual

Ativa

Inativa

Constatando que embora a IU seja um frequente problema de saúde entre os idosos, no Brasil as pesquisas ainda são realizadas com amostras reduzidas. Assim, faz-se necessária a elaboração de novos estudos com maior rigor metodológico que possibilite o planejamento de medidas de prevenção e tratamento adequados, visando um declínio dos gastos sanitários e redução do impacto dessa condição na saúde dos idosos (JEREZ et al., 2013).

Salienta-se que o presente estudo apresenta como limitação o número reduzido de pacientes. Outra limitação foi a não utilização de instrumentos objetivos para detectar o tipo de IU.

\section{CONCLUSÃO}

Conclui-se que na Clínica de Fisioterapia do Centro Universitário de Maringá o perfil dos pacientes com incontinência urinária caracteriza-se como sendo a maioria do sexo feminino, idosos, e com incontinência urinaria do tipo mista.

\section{REFERÊNCIAS}

ARAÚJO MP, PARMIGIANO TR, NEGRA LGD, TORELLI L, CARVALHO CG, WO L; MANITO, A. C. A.; GIRÃO, M. J. B. C.; SARTORI, A. S. F. Avaliação do assoalho pélvico de atletas: existe relação com a incontinência urinária? Revista Brasileira de Medicina do Esporte, v. 21, n. 6, p. 442-6, 2015. DOI: http://dx.doi.org/10.1590/1517-869220152106140065.

ARAÚJO, L. F.; ELES, M. A., NASCIMENTO, W. D.; SIQUEIRA, L. G.; GUSMÃO, M. S. Incontinência urinária em idosos. Revista Mineira de Educação Física, v. 5, p. 167-76, 2010. DISPONÍVEL

EM: http://www.revistamineiradeefi.ufv.br/artigos/arquivos/5451e57ebc3419f6779acdee3 50642a4.pdf

BORGES, J. B. R.; GUARISI, T.; CAMARGO, A. C. M.; BORGES, P. C. G. Correlação entre o estudo urodinâmico, a anamnese e os achados clínicos na abordagem de mulheres com incontinência urinária. Einstein., v. 8, n. 3, p. 437-43, 2010. DOI: http://dx.doi.org/10.1590/S1679-45082010AO1611. 
CARRARA, T.; ARAÚJO, M. S.; KINEQUITA, S. S.; NASCIMENTO, D. S.; ALDRIGHI, J. M. Avaliação do nível de orientação das mulheres no climatério sobre o papel da fisioterapia na prevenção e no tratamento da incontinência urinária. Revista Brasileira de Ciência e Envelhecimento, v. 9, n. 2, p. 171-9, 2012. DOI: http://dx.doi.org/10.5335/rbceh.2012.1574

CAVALCANTE, K. V. M.; SILVA, M. I. G. C.; BERNARDO, A. S. F.; SOUZA, D. E.; LIMA, T. C. G. C.; MAGALHÃES, A. G. Prevalência e fatores associados à incontinência urinária em mulheres idosas. Revista Brasileira de Promoção da Saúde, v. 27, n. 2, p. 216-23, 2014. DOI: http://dx.doi.org/10.5020/18061230.2014.p216

DEDICAÇÃO, A. C.; HADDAD, M.; SALDANHA, M. E. S.; DRIUSSO, P. Comparison of quality of life for different types of female urinary incontinence. Revista Brasileira de Fisioterapia, v. 13, n. 2, p. 112-6, 2009. DOI: http://dx.doi.org/10.1590/S141335552009005000014

EL-AZAB, A. S.; SHAABAN, O. M. Measuring the barriers against seeking consultation for urinary incontinence among Middle eastern women. BMC Women's Health, v. 10, n. 3, p. 1-6, 2010. DOI: http://dx.doi.org/10.1186/1472-6874-10-3

FIGUEIREDO, E. M.; LARA, J. O.; CRUZ, M. C.; QUINTÃO, D. M.; MONTEIRO, M. V. Perfil sociodemográfico e clínico de usuárias de serviço de fisioterapia uroginecológica da rede pública. Revista Brasileira de Fisioterapia, v. 12, p. 2, n. 136-42, 2008. DOI: http://dx.doi.org/10.1590/S1413-35552008000200010

JEREZ, J. R.; SOUZA, D. L. B.; LIMA, K. C. Incontinência urinária em idosos institucionalizados no Brasil: uma revisão integrativa. Revista Brasileira de Geriatria e Gerontologia, v. 16, n. 4, p. 865-79, 2013. DOI: http://dx.doi.org/10.1590/S1809-98232013000400020

JEREZ-ROIG, J.; SANTOS, M. M.; SOUZA, D. L. B.; AMARAL, F. L. J. S.; LIMA, K. C. Prevalence of Urinary Incontinence and Associated Factorsin Nursing Home Residents. Neurourology and Urodynamics. V. 35, p. 102-7, 2016. DOI: http://dx.doi.org/10.1002/nau.22675

KNORST, M. R.; RESENDE, T. L.; GOLDIM, J. R. Perfil clínico, qualidade de vida e sintomas depressivos de mulheres com incontinência urinária atendidas em hospitalescola. Revista Brasileira de Fisioterapia, v. 15, n. 2, p. 109-16, 2011. DOI: http://dx.doi.org/10.1590/S1413-35552011000200005

LEROY, L. S.; LOPES, M. H. B. M.; SHIMO, A. K. K. A. A incontinência urinária em mulheres e os aspectos raciais: uma revisão de literatura. Texto \& Contexto Enfermagem, v. 21, n. 3, p. 692-701, 2012. DOI: http://dx.doi.org/10.1590/S010407072012000300026.

LOPES, M. H. B. M.; MARTIN, H. F.; ORTEGA, N. R. S.; MARIN, H. F.; ORTEGA, N. R. S.; MASSAD, E.The use of expert systems on the differential diagnosis of urinary 
incontinence. Revista da Escola de Enfermagem da USP., v. 43, n. 3, p. 704-10, 2009. DOI: http://dx.doi.org/10.1590/S0080-62342009000300029.

MARQUES, L. P.; SCHNEIDER, I. J. C.; GIEH, M. W. C.; ANTES, D. L.; D'ÓRSI, E. Demographic, health conditions, and lifestyle factors associated with urinary incontinence in elderly from Florianópolis, Santa Catarina, Brazil. Revista Brasileira de Epidemiologia, v. 18, n. 3, p. 595-606, $2015 . \quad$ DOI: http://dx.doi.org/10.1590/1980-5497201500030006

MORRISROE, S. N.; RODRIGUEZ, I. V.; WANG, P. C.; SMITH, A. L.; SARKISIAN, A. C. Correlates of 1-year incidence of urinary incontinence in older Latino adults enrolled in a community-based physical activity trial. Journal of the American Geriatrics Society, v. 62, n. 4, p. 740-6, $2014 . \quad$ DOI: http://dx.doi.org/10.1111/jgs.12729.

NEKI, N. S. Urinary Incontinence in Elderly. Journal of Krishna Institute of Medical Sciences University, v. 5, n. 1, p. 5-13, 2016. DISPONÍVEL EM: http://jkimsu.com/jkimsu-vol5no1/JKIMSU,\%20Vol.\%205,\%20No.\%201,\%20Janarch\%202016\%20Page\%205-13.pdf

PARK, J.; HONG, G. R. S. Association of functional ability and benign prostatic hyperplasia with urinary incontinence in older korean men. International Neurourology Journal, v. 20, p. 137-42, $2016 . \quad$ DOI: http://dx.doi.org/10.5213/inj.1630432.216

PEDRO, A. F.; RIBEIRO, J.; SOLER, Z. A. G.; BUGDAN, A. P. Qualidade de vida de mulheres com incontinência urinária. Revista Eletrônica Saúde Mental, Álcool e Drogas, V. 7, n. 2, p.63-79, 2011. DOI: http://dx.doi.org/10.11606/issn.18066976.v7i2p63-70

SAMUELSSON, E.; ODEBERG, J.; STENZELIUS, K.; MOLANDER, U.; HAMMARSTROM, M.; FRANZEN, K.; ANDERSSON, G.; MIDLOY, P. Effect of pharmacological treatment for urinary incontinence in the elderly and frail elderly: a systematic review. Geriatrics \& Gerontology International, v. 15, n. 5, p. 521-34, 2015. DOI: http://dx.doi.org/10.1111/ggi.12451.

SANTOS, C. R. S.; SANTOS, V. L. C. G. Prevalência da incontinência urinária em amostra randomizada da população urbana de Pouso Alegre, Minas Gerais, Brasil. Revista Latino-Americana de Enfermagem, v. 18, n. 5, p. 903-10, 2010. DOI: http://dx.doi.org/10.1590/S0104-11692010000500010

SILAY, K.; AKINCI, S.; ULAS, A.; YALCIN, A.; SILAY, Y. S.; AKINCI, M. B.; DILEK, I.; YALCIN, B. Occult urinary incontinence in elderly women and its association with geriatric condition. European Review for Medical and Pharmacological Sciences, v. $20, \quad$ n. $3, \quad$ p. 447-51, 2016. DISPONÍVEL EM: http://www.europeanreview.org/article/10276 
SILVA, V. A.; D’ELBOUX, M. J. Fatores associados à incontinência urinária em idosos com critérios de fragilidade. Texto \& Contexto Enfermagem, v. 21, n. 2, p. 338-47, 2012. DOI: http://dx.doi.org/10.1590/S0104-07072012000200011

SMITH, A. P. Female urinary incontinence and wellbeing: results from a multinational survey. BMC Urology., v. 16, n. 22, p. 1-6, 2016. DOI: http://dx.doi.org/0.1186/s12894-016-0140-z.

SOUZA, J. O.; MASCARENHOAS, C. S.; NASCIMENTO, A. C. P.; GONÇALVES, A. S.; PEREIRA, L. C.; KURAIEM, M. A. P.; CRUZ, A. T. A prevalência de incontinência urinária em mulheres na pós menopausa. Revista Univap., v. 15, n. 37, p. 31-42, 2015.

http://revista.univap.br/index.php/revistaunivap/article/viewFile/288/262

EM:

SUMARDI, R.; MOCHTAR, C. A.; JUNIZAF, SANTOSO, B. I.; SETIATI, S.; NUHONNI, A. S.; TRIHONO, P. P.; RAHARDIO, H. E.; SVAHPUTRA, F. A. Prevalence of urinary incontinence, risk factors and its impact: multivariate analysis from Indonesian nationwide survey. Indonesian Journal of internal Medicine., v. 46, n. 3, p. 175-82, 2016. DISPONÍVEL EM: http://www.inaactamedica.org/archives/2014/25348179.pdf

TAMANINI, J. T. N.; DAMBROS, M.; D’ANCONA, C. A. L.; PALMA, P. C. R.; NETO JúNIOR, N. R. Validação para o português do "International Consultation on Incontinence Questionnaire - Short Form" (ICIQ-SF). Revista de Saúde Pública, v. 38, n. 3, p. 438-44, 2004. DOI: http://dx.doi.org/10.1590/S0034-89102004000300015

VITALE, S. G.; LAGANA, A. S.; RAPISARDA, A. M. Letter to the editor: Comment on" Simultaneous treatment of anterior vaginal wall prolapse and stress urinary incontinence by using transobturator four arms polypropylene mesh". Investigative and Clinical Urology, v. 57, n. 2, p. 150-1, 2016. DOI: http://dx.doi.org/10.4111/icu.2016.57.2.150

WAETJEN, E. L.; FENS, W. Y.; YE, J.; JOHNSON, W. O.; GREENDALE, G. A.; SAMPSELLE, C. M.; STERNFIELD, B.; HARLOW, S. D.; GOLD, E. B. Factors Associated with worsening and improving urinary incontinence across the menopausal transition. Obstetrics \& Gynecology, v. 111, n. 3, p. 667-77, 2008. DOI: http://dx.doi.org/10.1097/AOG.0b013e31816386ce 